\title{
La ecografia transvaginal es un buen método para descartar enfermedad endometrial
}

Transvaginal ultrasonography compared with endometrial biopsy for the detection of endometrial disease

N Engl J Med 1997; 337:1792-8. R. Langer, J Pierce, K O'Hanlan et al.

\begin{abstract}
Objetivo
Comparar la la sensibilidad y especificidad de la ecografía transvaginal (ETV) y la biopsia endometrial (BE) en el diagnóstico de enfermedad del endometrio en pacientes que reciben tratamiento con estrógeno solo, estrógeno y progesterona, o placebo durante 3 años.
\end{abstract}

\section{Diseño}

Seguimiento de una cohorte de pacientes asintomáticas posmenopáusicas del estudio PEPI (Postmenopausal Estrogen/ Progestin Interventions).

\section{Lugar}

El estudio PEPI fue un estudio multicéntrico realizado en Estados Unidos.

\section{Pacientes}

El PEPI incluyó 875 mujeres posmenopáusicas entre 45-64 años (media 59 \pm 4 ), con factores de riesgo para enfermedad cardiovascular con no más de 10 años (media $8 \pm 3$ ) desde su menopausia. Las mujeres con útero $(n=596)$ o sin útero fueron randomizadas para recibir placebo o uno de los siguientes tratamientos durante tres años: $0.625 \mathrm{mg} /$ día de estrógenos conjugados (EC) solos; EC más $2.5 \mathrm{mg}$ de acetato de medroxiprogesterona (MP)/día; EC más $10 \mathrm{mg}$ de MP/día, 12 días al mes; EC más $200 \mathrm{mg}$. de progesterona micronizada/día, 12 días al mes. Las mujeres con útero tenían BE normal al inicio del estudio.

\section{Descripción del tests de referencia (BE) y el test en estudio (ETV)} Se realizaron BE (test de referencia) en las visitas anuales clasificando los resultados como: normal; hiperplasia simple, compleja o atípica; y adenocarcinoma. La ETV fue realizada no más de $48 \mathrm{hs}$. previas a la biopsia. Se consideró como límite normal $4 \mathrm{~mm}$ de espesor y como valor umbral $9 \mathrm{~mm}$ en mujeres que recibían EC solos o tratamientos cíclicos. Si el espesor no era reportado no se lo analizaba.

\section{Medición de resultados principales}

El engrosamiento de la pared endometrial de $5 \mathrm{~mm}$ o más, y $9 \mathrm{~mm}$ o más fueron clasificados como anormales para evaluar la sensibilidad*, especificidad ${ }^{*}$ y valor predictivo positivo y negativo* de la ETV comparada con la BE. Como el objetivo era evaluar la ETV como estudio sustituto de la BE, la sensibilidad y los valores predictivos fueron recalculados luego de considerar negativos en vez de positivos los resultados de las ecografías insatisfactorios con grosor anormal. Esto se hizo así ya que se asumió que la ETV en estos casos no sería concluyente y se requeriría de todos modos la BE.
Del mismo modo, para calcular la especificidad se excluyeron las ecografías que no reportaban el grosor endometrial, ya que la ausencia de enfermedad no era confirmada.

\section{Resultados principales}

La ETV y la BE fueron realizadas en 448 mujeres (un total de 577 exámenes simultáneos). La pared endometrial fue $5 \mathrm{~mm}$ en el $45 \%$ de los casos, entre $5-10 \mathrm{~mm}$ en el $41 \%$, > $10 \mathrm{~mm}$ en el $12 \%$ y no medido en el $2 \%$.

Media del espesor de la pared endometrial y biopsia según el tratamiento

\begin{tabular}{|c|c|c|c|c|c|c|c|}
\hline \multirow[t]{2}{*}{ Tratamiento } & \multicolumn{2}{|c|}{$\begin{array}{l}\text { Media del } \\
\text { Espesor } \\
\text { Endometrial } \\
\text { en mm } \\
\text { (rango) }\end{array}$} & \multicolumn{2}{|c|}{ Biopsia } & \multicolumn{3}{|c|}{ Número de biopsias (\%) } \\
\hline & & Normal & $\begin{array}{l}\text { Hiperplasia } \\
\text { simple }\end{array}$ & $\begin{array}{l}\text { Hiperplasia } \\
\text { Compleja }\end{array}$ & $\begin{array}{l}\text { ia Hiperplasia } \\
\text { Atípica }\end{array}$ & $\begin{array}{l}\text { Adeno } \\
\text { carcinoma }\end{array}$ & Total \\
\hline Placebo & $3.8(1-15)$ & $142(98)$ & $2(1)$ & 0 & 0 & 1(1) & 145 \\
\hline EC & $11.7(1-26)$ & $44(66)$ & $16(24)$ & $5(7)$ & $2(3)$ & 0 & 67 \\
\hline $\begin{array}{l}\mathrm{EC}+\mathrm{MP} \\
\text { ciclico }\end{array}$ & $6.4(2-15)$ & $121(98)$ & 1(1) & 2(2) & 0 & 0 & 124 \\
\hline $\begin{array}{l}\text { EC + progest. } \\
\text { Micronizada }\end{array}$ & $6.4(1-4)$ & $100(99)$ & 1(1) & 0 & 0 & 0 & 101 \\
\hline $\begin{array}{l}\mathrm{EC}+\mathrm{MP} \\
\text { continuo }\end{array}$ & $4.5(1-17)$ & $122(100)$ & & 0 & 0 & $1(0)$ & 122 \\
\hline
\end{tabular}

Sensibilidad, Especificidad y valores predictivos*

\begin{tabular}{lll}
\hline $\begin{array}{l}\text { Espesor de pared } \\
\text { endometrial }>5 \mathrm{~mm}\end{array}$ & $\begin{array}{l}\text { Alguna } \\
\text { anormalidad }\end{array}$ & $\begin{array}{l}\text { Anormalidad } \\
\text { seria \# }\end{array}$ \\
\hline $\begin{array}{l}\text { Todas las Ecografias realizadas } \\
\text { Sensibilidad }\end{array}$ & $90(28 / 31) \# \#$ & $91(10 / 11)$ \\
\hline Especificidad & $48(259 / 538)$ & - \\
\hline Valor predictivo Positivo & $9(28 / 307)$ & $3(10 / 307)$ \\
\hline Valor Predictivo negativo & $99(259 / 261)$ & $100(261 / 261)$ \\
\hline Ecografias con resultados satisfactorios & $81(25 / 31)$ & $73(8 / 11)$ \\
\hline Sensibilidad & $46(247 / 538)$ & - \\
\hline Especificidad & $8(25 / 307)$ & $3(8 / 307)$ \\
\hline Valor predictivo Positivo & $95(247 / 261)$ & $95(249 / 261)$ \\
\hline Valor Predictivo negativo &
\end{tabular}

\# Incluye Hiperplasia compleja, Hiperplasia atípica y Adenocarcinoma

\#\# En paréntesis el Nro de examenes con los que se realizaron los cálculos.

Conclusiones

La ecografía transvaginal tiene un pobre valor predictivo positivo pero un alto valor predictivo negativo para detectar anormalidades endometriales serias en mujeres asintomáticas posmenopáusicas.

\section{COMENTARIO}

Una de las barreras de los médicos de atención primaria para prescribir terapia de reemplazo hormonal (TRH) se relaciona con las limitaciones que tienen para el manejo de los sangrados no esperados. Probablemente los médicos se sentirían más seguros con la prescripción de TRH si contaran con un método relativamente barato, incruento y que fuera capaz de descartar patología grave ante una mujer con sangrado no esperado. Este estudio fue diseñado para comparar la ecografía transvaginal con la biopsia endometrial como rastreo (screening) en mujeres asintomáticas con TRH (incluyó un grupo con placebo). Debemos recordar que el monitoreo endometrial (con ecografía, citología o histología) no está recomendado para mujeres que reciben TRH combinada dado que la prevalencia de cáncer de endometrio no aumenta en este grupo de pacientes; y por lo tanto debe reservarse para mujeres con TRH que presentan sangrados inesperados ${ }^{1}$. Se entiende como sangrado inesperado al que ocurre luego de seis meses si la paciente está con TRH continua, o al que es mayor en cantidad o duración que una menstruación normal, u ocurre fuera de los días 7 a 17 si la paciente recibe TRH cíclica. Debemos destacar que este estudio realizó ecografía transvaginal a todas las mujeres (que como se observa en la tabla 1 tenían una baja prevalencia de enfermedades graves) y se encontró un excelente VPN y un pobre VPP. También es importante destacar que la prevalencia de cáncer de endometrio no aumenta con el empleo de TRH combinada. Es decir que la presencia de una línea endometrial $<5 \mathrm{~mm}$ nos deja razonablemente tranquilos acerca de la ausencia de patología grave, pero que un número alto de mujeres con líneas $>5 \mathrm{~mm}$ se someterán a mayores estudios sin tener patología significativa. Si aplicarámos la ETV en mujeres con una mayor prevalencia de patología grave (como son las mujeres con metrorragia), el VPP mejoraría. 